\title{
Level-Set Function Approach to an Inverse Interface Problem
}

\author{
Kazufumi Ito* $\quad$ Karl Kunisch ${ }^{\dagger} \quad$ Zhilin $\mathrm{Li}^{\ddagger}$
}

\begin{abstract}
A model problem in electrical impedance tomography for the identification of unknown shapes from data in a narrow strip along the boundary of the domain is investigated. The representation of the shape of the boundary and its evolution during an iterative reconstruction process is achieved by the level set method. The shape derivatives of this problem involve the normal derivative of the potential along the unknown boundary. Hence an accurate resolution of its derivatives along the unknown interface is essential. It is obtained by the immersed interface method.
\end{abstract}

\section{Introduction}

In this paper we discuss the application of the level set function for identifying the unknown shape of an interface in a problem motivated by electrical tomography. For this purpose let $\mu$ denote the conductivity of the medium, $u$ the potential and $g$ the known boundary sources, and consider in a domain $\Omega$ with boundary $\partial \Omega$ the governing equation

$$
(\mu \nabla u, \nabla v)_{\Omega}=(g, v)_{\partial \Omega}, \quad \text { for } v \in H^{1}(\Omega) .
$$

We assume that $\Omega$ can be decomposed in two disjoint subdomains $\Omega^{+}$and $\Omega^{-}$and that the value of the conductivity is known on each of them. The problem consists in determining the interface $\Gamma$ between $\Omega^{+}$and $\Omega^{-}$from measurements $z$ of the potential $u$ along a thin layer along the boundary. The data can be considered to be obtained, for example, from boundary measurements by numerical extension into the interior of $\Omega$.

The representation of $\Gamma$ and its movement during an iteration process represents a severe difficulty. We propose to use the level set method with the zero level set giving the location of $\Gamma$. Level set method proposed in $[10,13]$ and many others in the literature is an extremely versatile tool for representing moving fronts in a variety of physical processes, involving flow phenomena, crystal growth and phase changes among others. In the context of inverse problems Santosa and coworkers in [12] and [9] applied level set techniques to deconvolution and inverse scattering problems.

\footnotetext{
${ }^{*}$ Center for Research in Scientific Computation \& Department of Mathematics, North Carolina State University, Raleigh, NC 27695, e-mail: kito@math.ncsu.edu

${ }^{\dagger}$ Insitute of Mathematics, University of Graz, Heinrichstrasse 36, A-8010 Graz, Austria, e-mail:karl.kunisch@ kfunigraz.ac.at

${ }^{\ddagger}$ Center for Research in Scientific Computation \& Department of Mathematics, North Carolina State University, Raleigh, NC 27695, e-mail: zhilin@math.ncsu.edu
} 
The approach we take is based on a least squares technique. The gradient of the cost functional is computed and utilized to advance the level set function, whose evolution is determined by a Hamilton Jacobi type equation. In our case the shape derivative involves the normal derivative of $u$ along $\Gamma$. As a consequence, in order to guarantee a numerically accurate shape derivative, the normal derivative of $u$ along $\Gamma$ has to be calculated with high accuracy. For this purpose the immersed interface method as developed in $[5,6,7]$ is used. It is known to be second order accurate along the boundary of interfaces. We believe that the success of identifying numerically some rather difficult shapes can primarily be attributed to the fact of high numerical accuracy of the potential and its derivatives along the interfaces.

The remainder of the paper is organized as follows. Section 2 contains the precise problem statement and a brief discussion of shape derivatives. The proposed numerical algorithm and several features of its implementation are described in Section 3. The fourth section is devoted to two numerical examples. In the Appendix the proof of the shape derivative of the solution to the state equation is given.

\section{Problem statement and shape derivative}

In this section we give the problem specification and a representation of the shape derivative that lends itself to a convenient use in the level set method. Let $\varphi=\varphi(t, x), t \geq 0, x \in R^{2}$ denote the level set function. It defines a family of interfaces $\Gamma_{t}$ by

$$
\Gamma_{t}=\left\{x \in R^{2}: \varphi(t, x)=0\right\}
$$

and domains

$$
\begin{aligned}
& \Omega_{t}^{+}=\{x \in \Omega: \varphi(t, x)>0\} \\
& \Omega_{t}^{-}=\{x \in \Omega: \varphi(t, x)<0\} .
\end{aligned}
$$

For convenience we fix the physical domain $\Omega$ to be $(-1,1) \times(-1,1)$ throughout. We consider the following interface problem for the potential function $u \in H^{1}(\Omega)$

$$
-\operatorname{div}(\mu(x) \operatorname{grad} u)=0
$$

with boundary condition

$$
\frac{\partial u}{\partial \nu}=g \quad \text { on } \partial \Omega
$$

where $\nu$ is the unit outer normal direction. The conductivity $\mu$ is supposed to be piecewise constant and is defined by

$$
\mu(x)= \begin{cases}\mu^{+} & x \in \Omega^{+} \\ \mu^{-} & x \in \Omega^{-} .\end{cases}
$$

The domain $\Omega^{-}$represents the inhomogeneity of the conducting medium. That is, if $\Omega^{-}=\emptyset$, then we have a homogeneously conducting medium. We assume that $\Omega^{-}$is the finite union of simply connected open sets in $\Omega$. Their boundary $\Gamma$ represents the interface between the two open domains 
$\Omega^{+}$and $\Omega^{-}$. It is assumed to be the union of closed $C^{2}$ curves. Let $\hat{\Omega}$ be the region of observation defined by

$$
\hat{\Omega}=\{x \in \Omega: \operatorname{dist}(x, \partial \Omega) \leq .2\},
$$

on which data of the potential function $u$ are assumed to be available. We further assume that the interface $\Gamma$ is strictly contained in $\hat{\Omega}$.

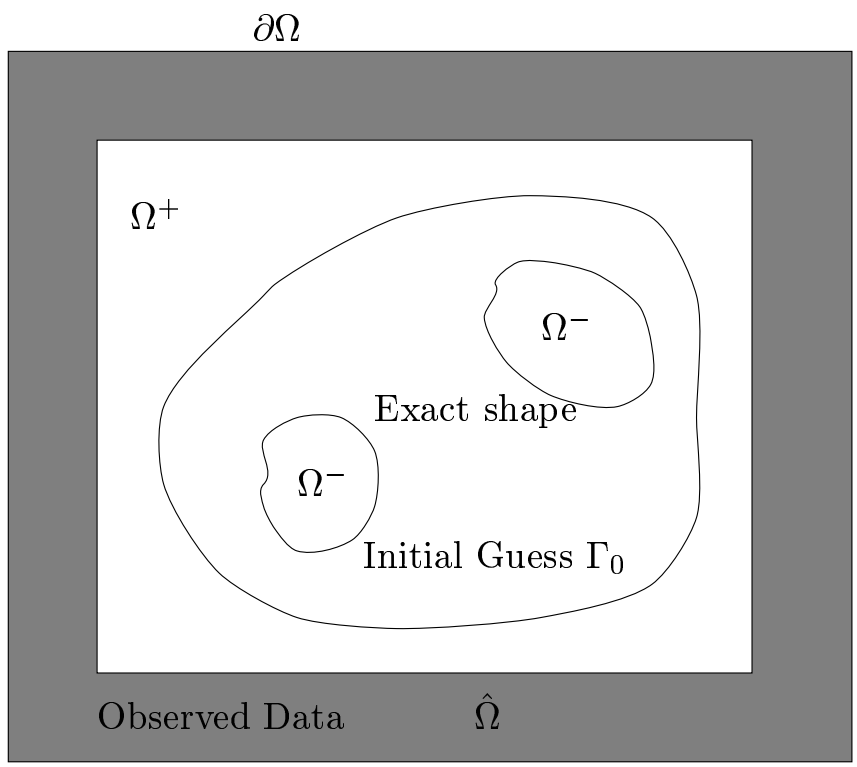

Figure 1: A diagram of the inverse problem of identifying the unknown shape from observed data taken from the shaded area.

We consider the inverse problem of identifying the unknown interface $\Gamma$ from the observation $z$ of $u$ on $\hat{\Omega}$. Given the interface $\Gamma$ let $u(\Gamma) \in H^{1}(\Omega) / R$ denote the solution to the boundary value problem $(2.2),(2.3)$. We formulate the least squares problem

$$
\min \quad J(\Gamma)=\int_{\hat{\Omega}} \frac{1}{2}|u(\Gamma)-z|^{2} d x+\epsilon \int_{\Gamma} 1 d s \quad \text { over } \Gamma \in Q_{a d},
$$

where $Q_{a d}$ is an admissible class of the interfaces and $\epsilon>0$.

Our numerical tests will be carried out for the case $\mu^{-}=\infty$ and thus, in case $\Omega^{-}$consists only of one connected component, the boundary value problem $(2.2),(2.3)$ reduces to

$$
-\Delta u=0 \quad \text { in } x \in \Omega^{+},
$$

with boundary conditions

$$
u=0 \quad \text { on } \Gamma \quad \text { and } \quad \frac{\partial u}{\partial \nu}=g \quad \text { on } \partial \Omega,
$$

where $\Gamma$ describes the shape that we are looking for. Note that $(2.5),(2.6)$ is meaningful without the assumption that $\Omega^{-}$consists of only one connected component. 
Next we consider the shape derivative of $u(\Gamma)$ and $J(\Gamma)$ with respect to $\Gamma$. First we discuss the shape derivative for the problem (2.5), (2.6). Let $\Gamma \in Q_{a d}$ be fixed and, for $|t|$ sufficiently small, let $\Omega_{t}=F_{t}\left(\Omega^{+}\right)$be the image of $\Omega^{+}$obtained by the mapping $F_{t}: R^{2} \rightarrow R^{2}$ defined as

$$
F_{t}\left(x_{1}, x_{2}\right)=\left(x_{1}, x_{2}\right)+t h\left(x_{1}, x_{2}\right) .
$$

For $\varphi \in H^{1}\left(\Omega^{+}\right)$and $\varphi_{t} \in H^{1}\left(\Omega_{t}\right)$ the material derivative of $\varphi$ for the field $h \in H^{1}\left(\Omega^{+}\right)^{2}$ is given by

$$
\dot{\varphi}(\mathbf{x})=\lim _{t \rightarrow 0} \frac{\varphi_{t}(\mathbf{x}+t \mathbf{h})-\varphi(\mathbf{x})}{t} \quad \text { for } \mathbf{x} \in \Omega^{+} .
$$

If $\dot{\varphi}(\mathbf{x})$ has a regular extension to a neighborhood of $\bar{\Omega}_{t}$, then

$$
\varphi^{\prime}(\mathbf{x})=\lim _{t \rightarrow 0} \frac{\varphi_{t}(\mathbf{x})-\varphi(\mathbf{x})}{t}=\dot{u}(\mathbf{x})-\mathbf{h}(\mathbf{x}) \cdot \nabla u(\mathbf{x}), \quad \mathbf{x} \in \Omega^{+}
$$

is called the shape derivative of $u$. These notions are standard in the theory of shape optimization, we refer to [3], [11] and [14], for example.

It can be shown that the shape derivative $u^{\prime}$ of the solution $u$ to (2.5), (2.6) is given by

$$
-\Delta u^{\prime}=0
$$

with boundary conditions

$$
u^{\prime}+\frac{\partial u}{\partial \nu}(\nu \cdot h)=0 \quad \text { on } \Gamma \quad \text { and } \quad \frac{\partial u^{\prime}}{\partial \nu}=0 \quad \text { on } \partial \Omega
$$

For a proof we refer to the above mentioned references. For the convenience of the reader we also provide a complete proof in the Appendix.

Let $p \in H^{1}\left(\Omega^{+}\right)$be the adjoint function satisfying

$$
-\Delta p=(u-z) \chi_{\hat{\Omega}},
$$

with boundary condition

$$
p=0 \text { on } \Gamma \quad \text { and } \quad \frac{\partial p}{\partial \nu}=0 \text { on } \partial \Omega,
$$

where $\chi_{\hat{\Omega}}$ is the indicator function of the domain $\hat{\Omega}$. Then for $\epsilon=0$

$$
\begin{gathered}
\lim _{t \rightarrow 0} \frac{J\left(\Gamma_{t}\right)-J(\Gamma)}{t}=\int_{\hat{\Omega}}\left(u(\mathbf{x})-z(\mathbf{x}), u^{\prime}(\mathbf{x})\right) d x \\
=\left\langle-\Delta p, u^{\prime}\right\rangle=\int_{\Gamma}(\nabla u \cdot \nabla p)(\nu \cdot \mathbf{h}(\mathbf{x})) d s
\end{gathered}
$$

where we used the fact that the tangential derivatives $u_{\tau}=p_{\tau}=0$. Thus the steepest descent direction $\vec{V}$ of $J$ is given by

$$
\vec{V}(\mathbf{x})=-(\nabla u \cdot \nabla p) \nu \quad \text { on } \Gamma
$$


Since for the normal direction we have $-\nu=\frac{\nabla \varphi}{|\nabla \varphi|}$ at $\Gamma$, we obtain the Hamilton-Jacobi equation for the level set function $\varphi=\varphi(t, \mathbf{x})$ :

$$
\varphi_{t}+V(t, \mathbf{x})|\nabla \varphi|=0
$$

where $V(t, \mathbf{x}) \nu$ is defined by

$$
V(t, \mathbf{x})=\nabla u \cdot \nabla p \quad \text { on } \Gamma
$$

and $(u, p) \in H_{0}^{1}\left(\Omega_{t}^{+}\right) \times H^{1}\left(\Omega_{t}^{+}\right)$satisfy

$$
-\Delta u=0 \quad \text { and } \quad-\Delta p=(u-z) \chi_{\hat{\Omega}}
$$

with boundary conditions

$$
u=p=0 \text { on } \Gamma_{t} \quad \text { and } \quad \frac{\partial u}{\partial \nu}=g, \quad \frac{\partial p}{\partial \nu}=0 \text { on } \partial \Omega .
$$

In case $\epsilon$ is positive the additive term $\epsilon \kappa$ has to be added to $V$, where $\kappa$ denotes the curvature along $\Gamma$.

\section{Numerical algorithm}

Given data on the domain $\hat{\Omega}$, and $g$ along $\partial \Omega$, the algorithm to identify the unknown $\Gamma$ inside the domain $\Omega$ is outlined below.

- Set an initial level set function $\varphi(x, y)$ as initial guess of the envelope of the unknown shape $\Gamma_{0}=\{\varphi(x, y)=0\}$.

For $k=0, k=1, \cdots$, do the following until the algorithm converges,

- Solve the Laplace equation (2.5)-(2.6) in $\Omega_{k}^{+}$for $u\left(\Gamma_{k}\right)$, where we use $k$ to indicate the quantities in the $k$-th step.

- Compute the difference of the computed solution with the observed data, i.e. $\left(u\left(\Gamma_{k}\right)-z\right) \chi_{\hat{\Omega}}$.

- Solve the Poisson equation (2.11)-(2.12) in $\Omega_{k}^{+}$.

- Evaluate the normal velocity $\Gamma_{k}$ using weighted least squares interpolation to get

$$
V_{k}=\nabla p_{k} \cdot \nabla u_{k}+\epsilon \kappa\left(\Gamma_{k}\right)
$$

where $\epsilon$ is a regularization parameter, and $\kappa\left(\Gamma_{k}\right)$ is the curvature of the boundary of the shape $\Gamma_{k}$.

- Extend the velocity $V_{k}$ to a computational tube $\left|\varphi^{k}\right| \leq \delta$, where $\delta$ is the width of the tube. 
- Update the level set function by solving the Hamilton-Jacobi equation $\varphi_{t}^{k}+V_{k}\left|\nabla \varphi^{k}\right|=0$ for $\varphi^{k}$ using

$$
\frac{\varphi^{k+1}-\varphi^{k}}{\Delta t}+V_{k}\left|\nabla \varphi^{k}\right|=0
$$

where $\left|\nabla \varphi^{k}\right|$ is evaluated using a WENO (weighted essential non-oscillatory) scheme, and $\Delta t$ is chosen as

$$
\Delta t=\frac{h}{2\left\|V_{k}\right\|_{\infty}}
$$

- Check convergence. Options include repeating the process, stopping if convergence criteria are satisfied, and starting from another initial level curve. Update $\Gamma_{k}=\left\{\varphi^{k}(x, y)=0\right\}$ and $\Omega_{k}$.

Several issues of implementation of the algorithm are discussed in the following sections.

\subsection{Fast immersed interface method for Poisson equations on irregular domains}

A Poisson solver based on the fast immersed interface method (IIM) developed in [7] and modified in [4] was used. The main idea of the IIM is to extend the Poisson equation from $\Omega^{+}$to the entire rectangular domain. This procedure allows the use of fast Poisson solvers on a fixed Cartesian grid independent of the shape of the irregular domain.

To briefly explain the procedure we extend the source term in the Poisson equation by zero into $\Omega^{-}$. We require the normal derivative of the solution $\psi$ to be continuous across the immersed boundary $\Gamma$ of the irregular part of the domain. The solution itself is allowed to have a finite jump $w$. In the language of potential theory this requirement is equivalent to the introduction of a double-layer source on $\Gamma$. This extension leads to the following interface problem,

$$
\begin{aligned}
& \Delta \psi= \begin{cases}f(x, y) & \text { if }(x, y) \in \Omega^{+}, \\
0 & \text { if }(x, y) \in \Omega^{-},\end{cases} \\
& {\left[\psi_{n}\right]=0, \quad \text { on } \Gamma \text {, }} \\
& {[\psi]=w, \quad \text { on } \Gamma,} \\
& \frac{\partial \psi}{\partial n}=g(x, y), \quad \text { on } \partial \Omega,
\end{aligned}
$$

where [.] denotes the jump across $\Gamma$. We choose $w$ so that the solution $\psi$ of (3.20) satisfies homogeneous Dirichlet boundary condition

$$
\psi^{+}=0, \quad \text { on } \Gamma,
$$

where $\psi^{+}$is the limiting value of the solution on the boundary from within $\Omega^{+}$. Concerning the well-posedness of (3.20) and (3.21) we refer to [2].

To numerically compute the solution of (3.20)-(3.21) for $\psi$ and $v$, we use the standard discrete five point stencil plus a correction term at irregular grid points. Irregular grid points $\left(x_{i}, y_{j}\right)$ are 
those at which the level set function has a different sign from at least one pair of its neighbors. We denote the vector of the discretized values of $\psi$ on $\Omega$ by $\Psi$, and the vector of the discretized values of the jump $w$ at the projections of the irregular grid points that lie inside $\Omega^{+}$by $W$. On an $N \times N$ grid, the number of components $N_{\Psi}$ of $\Psi$ is approximately $N^{2}$, while the number of components $N_{W}$ of $W$ is on the order of $N$. Using the IIM [5,6] to discretize the interface problem (3.20), we get a system of $N_{\Psi}$ linear equations of the form

$$
A \Psi+B W=F_{1},
$$

where $A$ is the discrete Laplacian matrix, $B$ is a sparse matrix, and the vector $F_{1}$ is a source term depending on $f$ and $g$ and appropriately modified at the irregular grid points. Discretizing the Dirichlet boundary condition in (3.20) and (3.21) on the immersed boundary $\Gamma$, we get a system of $N_{W}$ linear equations of the form

$$
C \Psi+D W=F_{2} .
$$

Thus we obtain the following system of equations for the solution $\Psi$ and the jump $W$ on the boundary,

$$
\left[\begin{array}{ll}
A & B \\
C & D
\end{array}\right]\left[\begin{array}{c}
\Psi \\
W
\end{array}\right]=\left[\begin{array}{c}
F_{1} \\
F_{2}
\end{array}\right] .
$$

The Schur complement of (3.24) is

$$
\left(D-C A^{-1} B\right) W=G
$$

where

$$
G=F_{2}-C A^{-1} F_{1}
$$

Equation (3.25) for $W$ is a much smaller system than equation (3.24) for $(\Psi, W)$. An efficient choice to solve (3.25) is the generalized minimum residue (GMRES) method. Each iteration of the GMRES method involves one matrix-vector multiplication by $A^{-1}$, which we compute by means of a call to a fast Poisson solver for (3.22) with a specified jump $W$ in the solution. Each iteration also involves one call to the interpolation scheme to evaluate the residual $R=C \Psi+D W-F_{2}$ of the boundary condition (3.23) for the iterate.

This Poisson solver for irregular domains is second order accurate. The number of calls on the rectangular domain is equal to the number of GMRES iterations, and is almost independent of the mesh size, however, it may depend on the geometry of the domain.

In our implementation, the matrices and vectors are never explicitly formed. The fast solvers with examples for Poisson/Helmholtz equations on irregular domains are available to the public through anonymous ftp at ftp ftp.ncsu.edu under the directory $/$ pub/math/zhilin/Packages.

\subsection{Evaluation of gradient vectors at irregular points}

In order to use the level set method, we need the normal velocity at all grid points in the computational tube given by $|\varphi| \leq \delta$. In the computations we usually take $\delta$ between $1.6 h \sim 5 h$, where $h$ denotes the meshsize. 
The approach that we take is to find the normal velocities at the irregular points and then to extend them into the computational tube. To get robust and stable gradients at an irregular grid point $\left(x_{k}, y_{l}\right)$ where the level set function changes sign in the standard five point stencil, we use the weighted least squares interpolation, for example,

$$
u_{x}\left(x_{k}, y_{l}\right) \approx \sum_{i, j} \beta_{i j} u_{k+i, l+j}
$$

where the summation is taken over the set

$$
i^{2}+j^{2} \leq\left\{\operatorname{int}\left(\frac{\delta}{h}\right)\right\}^{2}, \quad\left(x_{k+i}, y_{l+j}\right) \in \Omega^{+} .
$$

The coefficients are the least squares solution of the following set of equations:

$$
\begin{aligned}
\sum_{i, j} \beta_{i j} & =0, & & \sum_{i, j} \beta_{i j}\left(x_{k+i}-x_{k}\right)=1, \\
\sum_{i, j} \beta_{i j}\left(y_{l+j}-y_{l}\right) & =0, & & \sum_{i, j} \beta_{i j}\left(x_{k+i}-x_{k}\right)^{2} / 2=0, \\
\sum_{i, j} \beta_{i j}\left(x_{k+i}-x_{k}\right)\left(y_{l+j}-y_{l}\right) & =0, & & \sum_{i, j} \beta_{i j}\left(y_{l+j}-y_{l}\right)^{2} / 2=0 .
\end{aligned}
$$

The same interpolation scheme is also used to evaluate $p_{x}$ on the irregular grid points.

\subsection{Extension of the velocity}

After having evaluated the normal velocities at the irregular grid points they need to be extended to all grid points inside the computational tube surrounding the boundary of the shape. This is done through an upwind scheme along the normal direction away from the interface

$$
V_{t} \pm \nabla V \cdot \frac{\nabla \varphi}{|\nabla \varphi|}=0
$$

where $V$ is the normal velocity, see $[1,8]$ and the references therein. The sign is determined from the normal direction of the level set function. In Figure 2 (a) we give a plot of the normal velocity at irregular grid points, while Figure 2 (b) depicts the normal velocity after extension, where $\Gamma$ is an ellipse.

Technically the motion of the free boundary is uniquely determined by the normal velocity of the boundary. By constructing the normal velocity in a neighborhood of the interface, we can avoid unnecessary numerical difficulties when updating the level set function. Therefore it seems that a first order method is sufficient since the velocity at irregular grid points remains unchanged. The velocity away from interface does not have much effect on the position of the interface rather the behavior of the level set function. The level set function then is preconditioned every few steps $[1,4]$ so that $|\nabla \varphi| \approx 1$ near the front by the re-initialization process. However, we should point it out that there are alternative approaches such as the fast marching method [4] to extend the velocity off the front. 
(a)

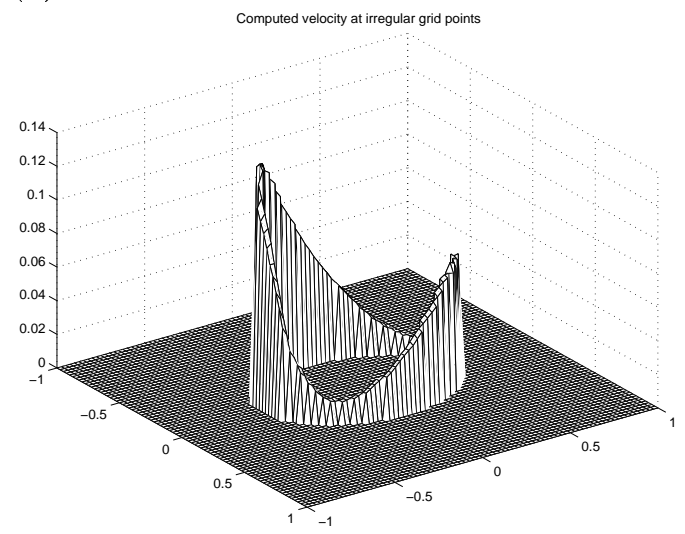

(b)

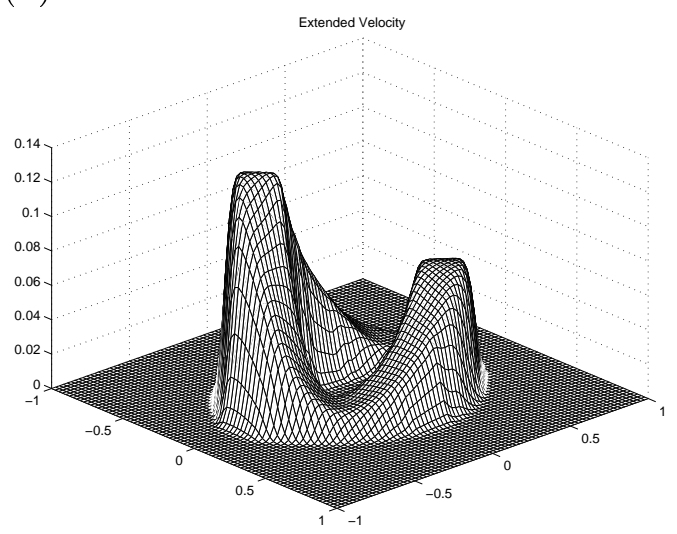

Figure 2: Velocity extension. (a): Computed velocity at irregular grid points. (b): Velocity field in a tube after the extension.

\subsection{Identifying several minima}

For the minimization problem (2.4), the solution may not be unique. There can be several local and global minima. Starting with an initial guess that contains all possible expected shapes, the algorithm typically finds quickly an envelope of all shapes representing the minima. To determine possible further minima inside of this envelope the following algorithm was successful in some situations.

Denote the level set function of the first equilibrium, the envelope, by $\varphi_{e}(x, y)$ and set $\varphi_{\min }=$ $\min \{\varphi(x, y)\}$. Then the algorithm is repeated with the initial level set function obtained by shifting $\varphi_{e}(x, y)$. That is, given a step size $d \varphi=\left|\varphi_{\text {min }}\right| / L$, we use the level set functions

$$
\varphi_{0}=\varphi_{e}+k d \varphi, \quad k=1,2, L-1
$$

as initial guesses to repeat the algorithm $L-1$ times. In this way, we can search for possible multiple local or global minima.

\section{Numerical example}

In this section, we provide some numerical examples. The domain is $[-1,1] \times[-1,1]$ unless specified otherwise. The boundary condition is taken as $g=1$ along $x=-1$ and $y=-1$ and $g=-1$ along $x=1$ and $y=1$. The width of the observed data is 0.2 . All the simulations were conducted using Sun workstations. Most of simulations are done within hours depending on the mesh size.

First we test our method for a single object in the domain. The exact shape is a skinny ellipse

$$
\frac{x^{2}}{0.07^{2}}+\frac{y^{2}}{0.5^{2}}=1
$$

which is the solid line in Fig. 3 (a) and (b). Fig. 3 (a) gives the result based on a 80 by 80 grid and Fig. 3 (b) depicts the result computed using a 160 by 160 grid. 
In Fig. 4, we plot the residual of the computed and exact potential defined as

$$
E=\sum_{i, j,(i, j) \in \bar{\Omega}_{h}}\left\|u_{o b, i j}-U_{i j}\right\| .
$$

The initial guess is a circle that surrounds the exact shape. The circle shrinks quickly in the radial direction to a small circle, see the rapid decline of the residue in Fig. 4. Then it gradually extends in the direction of the major axis and expands in the direction of the minor axis slowly, see also Fig. 4 after about 100 steps. The average of number of GMRES iterations is between 4 and 10 . Each iteration requires 2 calls to the Poisson solver, one for the primal variable $u$, one for the adjoint variable $p$.

We find the algorithm is very efficient for single objects in the domain.

\subsection{Noise analysis}

In practice, the observed data are corrupted by noise. We therefore also tested our algorithm with data given by $z_{i, j}=u\left(\Gamma^{*}\right)_{i, j}+\delta_{i, j}$, where $\Gamma^{*}$ denotes the "true" interface and $\delta_{i, j}$ is chosen as uniformly distributed random noise.

In the example that we present here the infinity norm of the perturbation in the case with high noise is $\|\delta\|_{\infty}=48.8332$ and the relative perturbation is $\|\delta\|_{\infty} /\left\|u_{\Gamma^{*}}\right\|_{\infty}=0.6768$. In Fig. 5 (a) we show the unperturbed data, while Fig. 5 (b) gives the observed data with noise of $68 \%$. Fig. 6 (b) is the computed result using data with $68 \%$ noise. In Fig. 6 (a) we can compare to the case where the noise level is only $17 \%$. While $\epsilon$ was chosen to be $10^{-6}$ in the noise free examples, we chose it to be $10^{-4}$ in the cases containing noise. We can summarize that our method also works well in the presence of noise.
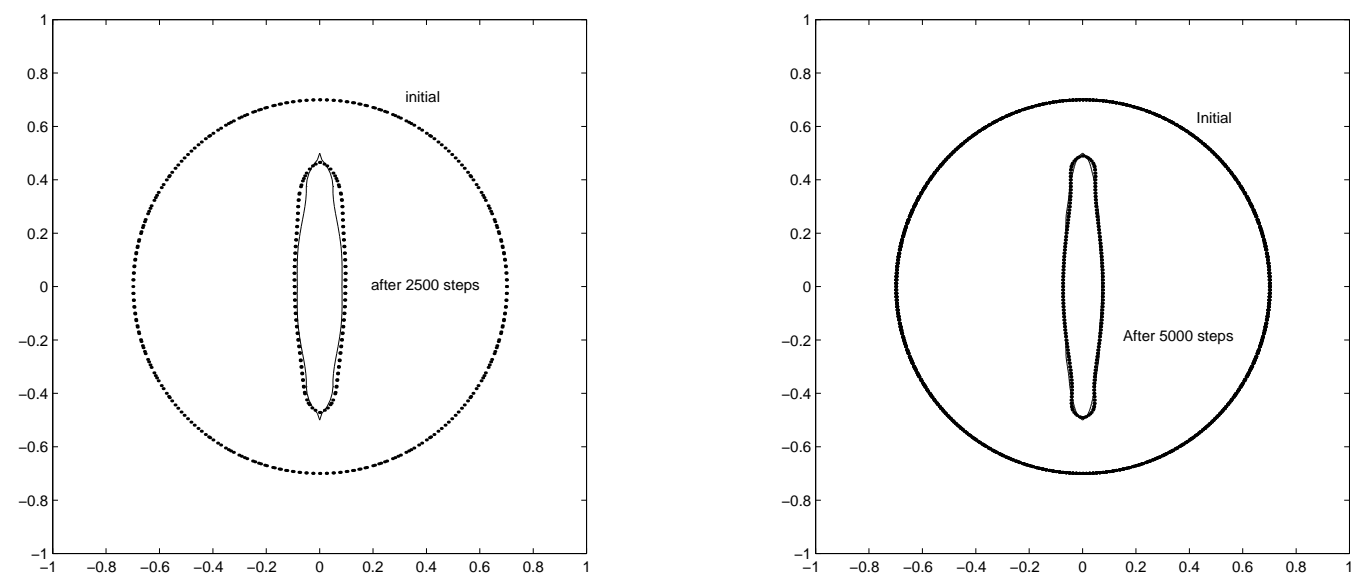

Figure 3: Computed shape with time step size $\Delta t=\frac{h}{2}, \epsilon=10^{-6}$. The initial guess is the big circle of radius 0.6 centered at the origin. (a) The result obtained using an 80 by 80 grid. (b) Result obtained using a 160 by 160 grid. 


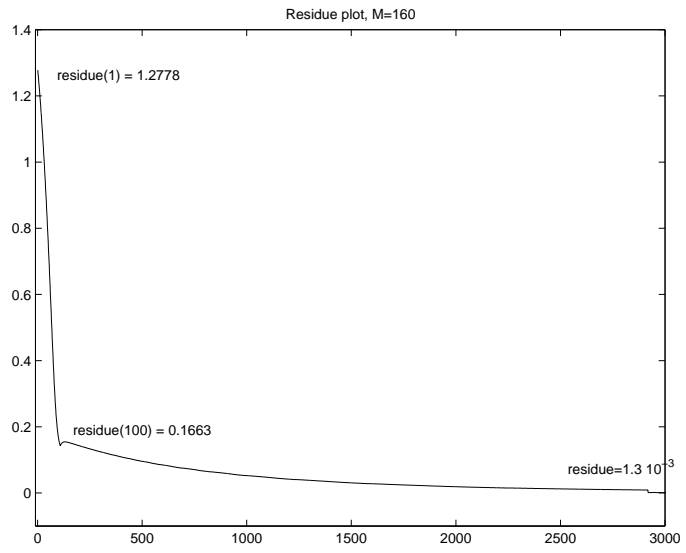

Figure 4: Plot of the residual versus the number of evolutions.
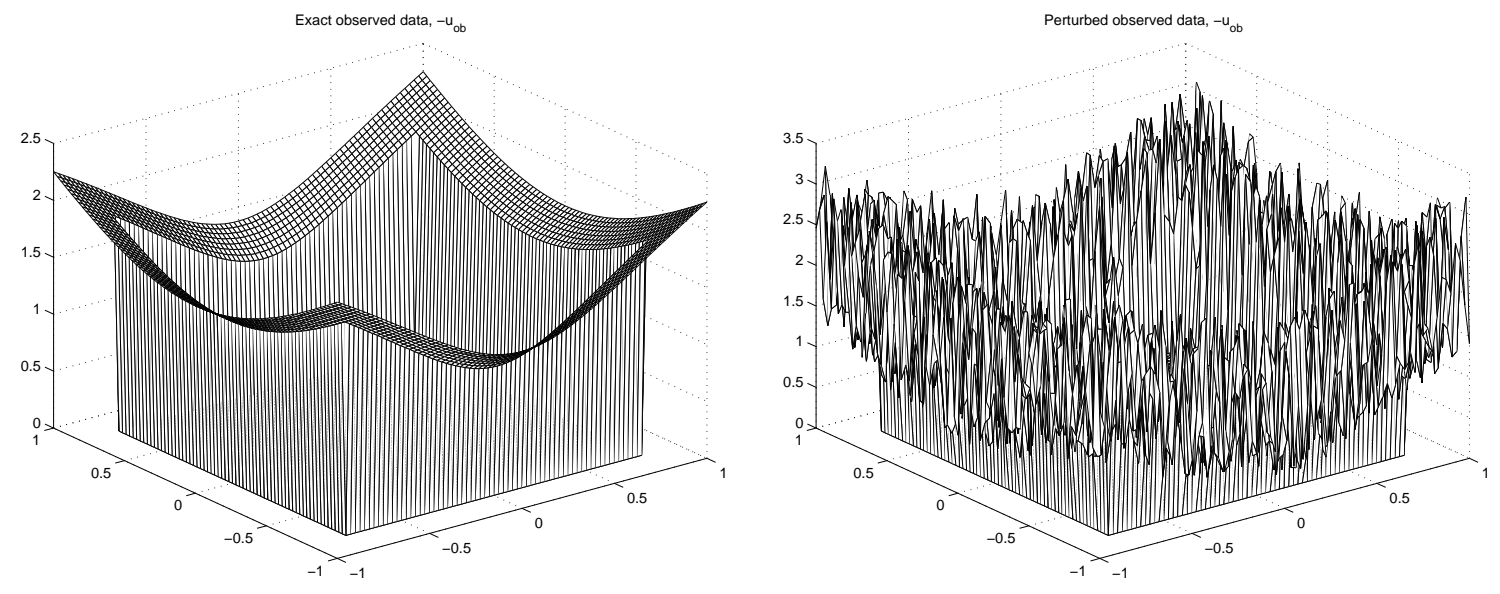

Figure 5: Exact data corresponding the exact shape. (b) The observed data with $68 \%$ relative uniform noise level. 

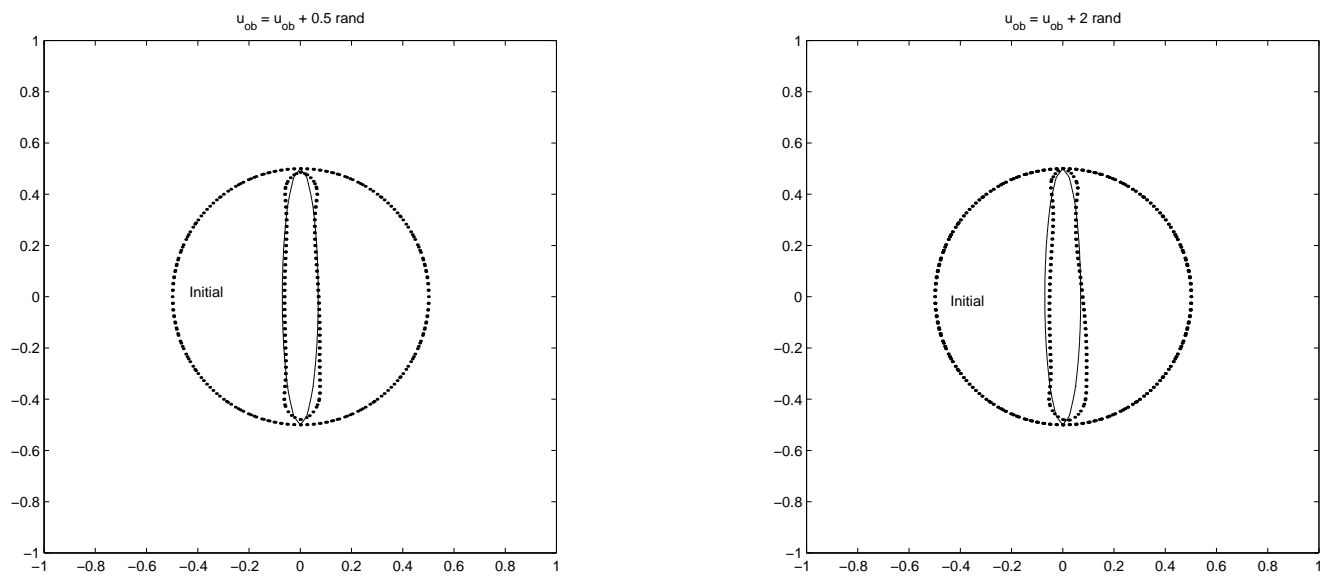

Figure 6: Computed results with noise data. (a) With 17\% uniformly distributed relative noise level. (b) With $68 \%$ uniformly distributed relative noise level.

\subsection{An example with two objects}

Now we consider an example that has two objects in the domain. The exact shape is composed of the following two closed curves:

$$
\begin{aligned}
& \frac{(x+0.35)^{2}}{0.25^{2}}+\frac{(y+0.35)^{2}}{0.3^{2}}=1 \\
& \frac{(x-0.35)^{2}}{0.2^{2}}+\frac{(y-0.35)^{2}}{0.2^{2}}=1 .
\end{aligned}
$$

The algorithm soon produces an envelope of the two objects and it can be checked numerically that the envelope is indeed a local minimum of the residual. Now we proceed as explained in Section 3.4 and denote the level set function corresponding to this local minimum by $\varphi_{\text {env }}$. Then we use

$$
\varphi_{\text {env }}+k d \varphi, \quad k=1,2, \cdots
$$

as new initial guesses to see if we can find further local extrema. In our example, $d \varphi=0.03$. Fig. 7 gives the plot of the computed objects with different values for $k$. In Fig. 7 (a) the convergence result to the envelope from an initial guess chosen as a circle with radius 0.7 centered at the origin is given. For the result in Fig. 7 (b), the initial level set was perturbed by the quantity 0.06 resulting in the smaller one of the two ellipses. The iterations of the algorithm were attracted back to the envelope and it appears that the envelope is a stable local extremum. In Fig. 7 (c), the zero level set was moved by the quantity 0.09 , and the zero level set converges with reasonable accuracy to the desired shape, consisting of two objects. In Fig. 7 (c), the zero level set was moved by the quantity 0.18 resulting in an initial level set consisting of two small closed curves. The zero level curves produced by the algorithm again approach the envelope as shown in Fig. 7 (a). 

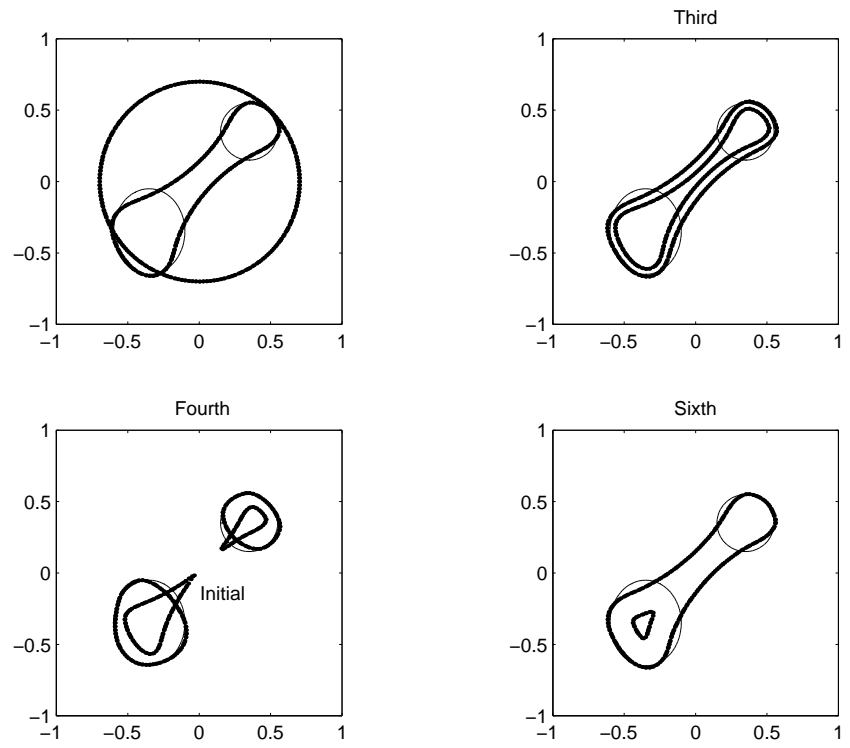

Figure 7: Computed results using automatic adjusting of the initial zero level set function with an 80 by 80 grid. (a) The initial guess is the circle $r=0.7$. (b) The initial level set is $\varphi=\varphi_{\text {env }}+0.06$, where $\varphi_{\text {env }}$ is the level set corresponding to the envelope. (c) the initial level set is $\varphi=\varphi_{\text {env }}+0.09$. (d) initial level set is $\varphi=\varphi_{e n v}+0.18$.

\section{Conclusions}

In this paper we considered a model problem from electrical tomograpy in which it is required to identify unknown conductivities from near-boundary measurements of the potential. It is assumed that the value of the conductivity is known in subregions whose boundaries are unknown. The level set function technique was shown to be succesfull to identify the unknown boundary shapes. It was further established that the immersed interface method provides an efficient tool for computing the state and the adjoint equations on domains which change during the course of the iterations, as well as for accurate representation of the normal derivative along the boundary, which is required for the computation of the shape derivative. The proposed algorithm was shown to be numerically effective for single and two component shapes.

\section{Acknowledgements}

The first and the third authors were partially supported by the ARO grant 39676-MA. The first author was also supported by MURI-AFOSR grant F49620-95-1-0447. The second author was supported by the Fonds zur Förderung der wissenschaftlichen Forschung under SFB 03 Optimierung und Kontrolle. The third author was also supported by an NSF grant DMS0073403. 


\section{References}

[1] S. Chen, B. Merriman, P.Smereka, and S. Osher. A fast level set based algorithm for Stefan problems. J. Comput. Phys., 135:8-29, 1997.

[2] Z. Chen and J. Zou. Finite element methods and their convergence for elliptic and parabolic interface problems. Numer. Math., 79:175-202, 1998.

[3] J. Haslinger and P. Neittanmaaki. Finite Element Approximation for Optimal Shape Design: Theory and Applications. J. Wiley, Chichester, 1988.

[4] T. Hou, Z. Li, S. Osher, and H. Zhao. A hybrid method for moving interface problems with application to the Hele-Shaw flow. J. Comput. Phys., 134:236-252, 1997.

[5] R. J. LeVeque and Z. Li. The immersed interface method for elliptic equations with discontinuous coefficients and singular sources. SIAM J. Numer. Anal., 31:1019-1044, 1994.

[6] Z. Li. The Immersed Interface Method - A Numerical Approach for Partial Differential Equations with Interfaces. PhD thesis, University of Washington, 1994.

[7] Z. Li. A fast iterative algorithm for elliptic interface problems. SIAM J. Numer. Anal., 35:230-254, 1998.

[8] Z. Li and B. Soni. Fast and accurate numerical approaches for Stefan problems and crystal growth. Numerical Heat Transfer, B: Fundamentals, 35:461-484, 1999.

[9] A. Litman, D. Lesselier, and F. Santosa. Reconstruction of a 2-d binary obstacle by controlled evolution of a level-set. Inverse Problems, 14:685-706, 1998.

[10] S. Osher and J.A. Sethian. Fronts propagating with curvature-dependent speed: Algorithms based on Hamilton-Jacobi formulations. J. Comput. Phys., 79:12-49, 1988.

[11] O. Pironneau. Optimal Shape Design for Elliptic Systems. Springer-Verlag, Heidelberg, 1984.

[12] F. Santosa. A level-set approach for inverse problems involving obstacles. ESAIM: COCV, 1:17-33, 1996.

[13] J. A. Sethian. Level Set Methods and Fast Marching methods. Cambridge University Press, 2nd edition,1999.

[14] J. Sokolowski and J.P. Zolesio. Introduction to Shape Optimization. Springer-Verlag, 1991. 


\section{Appendix}

We provide a proof for the shape derivative of the solution to (2.5), (2.6) based on the method of mappings. For $\varphi \in H^{1}(\Omega)$ and $\varphi_{t} \in H^{1}\left(\Omega_{t}\right)$ let us define

$$
\varphi^{t}=\varphi_{t} \circ F_{t}
$$

Define

$$
I_{t}=\operatorname{det}\left(D F_{t}\right) \quad \text { and } \quad A_{t}=\left(D F_{t}^{-1}\right)^{*}\left(D F_{t}^{-1}\right) I_{t}
$$

where $*$ denotes the transpose of a matrix and $D F_{t}$ is the Jacobian of $F_{t}$. It is then easy to verify that

$$
\begin{aligned}
& \left.\frac{d}{d t} F_{t}\right|_{t=0}=h,\left.\frac{d}{d t}\left(D F_{t}\right)\right|_{t=0}=D h,\left.\frac{d}{d t}\left(D F_{t}^{-1}\right)\right|_{t=0}=-(D h)^{*} \\
& \left.\frac{d}{d t} I_{t}\right|_{t=0}=\operatorname{div} h \quad \text { and }\left.\quad \frac{d}{d t} A_{t}\right|_{t=0}=\operatorname{div} h I-\left((D h)^{*}+D h\right) .
\end{aligned}
$$

Moreover we have

Lemma A.1 Let

$$
E_{t}=\int_{\Omega_{t}} \varphi_{t} d x_{t}, \quad \varphi_{t} \in H^{1}\left(\Omega_{t}\right)
$$

Then

$$
\dot{E}=\left.\frac{d}{d t} E_{t}\right|_{t=0}=\int_{\Omega} \dot{\varphi}+\varphi \operatorname{div} h d x=\int_{\Omega} \varphi^{\prime}+\operatorname{div}(h \varphi) d x
$$

Proof: Using Fubini's theorem we obtain

$$
E_{t}=\int_{\Omega} \varphi^{t} I_{t} d x
$$

By differentiating $E_{t}$ with respect to $t$ we obtain

$$
\frac{d}{d t} E_{t}=\int_{\Omega} \frac{d}{d t} I_{t} \varphi^{t}+I_{t} \frac{d}{d t} \varphi^{t} d x
$$

Since $I_{0}=1$ and $\left.\frac{d}{d t} I_{t}\right|_{t=0}=\operatorname{div} h$, (A.3) follows by setting $t=0$.

Now we turn to (2.5), (2.6) and derive the shape derivative of $u$. Note that $\nabla \varphi_{t}=\left(D F_{t}^{-1}\right) \nabla \varphi^{t}$. Thus, the boundary value problem

$$
-\Delta u=0 \quad \text { in } \Omega_{t}
$$

with $u=0$ on $\Gamma_{t}$ and $\frac{\partial u}{\partial \nu}=g$ on $\Gamma_{0}$ can equivalently be written as: $u^{t} \in H_{\Gamma}^{1}\left(\Omega^{+}\right)$satisfies

$$
\left(A_{t} \nabla u^{t}, \nabla \phi\right)_{L^{2}\left(\Omega^{+}\right)}=(g, \phi)_{L^{2}\left(\Gamma_{0}\right)}
$$


for $\phi \in H_{\Gamma}^{1}\left(\Omega^{+}\right)$, where $H_{\Gamma}^{1}\left(\Omega^{+}\right)=\left\{\phi \in H^{1}\left(\Omega^{+}\right): \phi=0\right.$ on $\left.\Gamma\right\}$.

Since $F_{t}, A_{t}, I_{t}$ are continuously differentiable in $t$ and Lipschitz in $x$ it follows from the implicit function theory that for $|t|$ sufficiently small (A.4) has a unique solution $u^{t} \in H_{\Gamma}^{1}\left(\Omega^{+}\right) \cap H^{2}\left(\Omega^{+}\right)$ and

$$
\lim _{t \rightarrow 0} \frac{u^{t}-u}{t}=\dot{u}
$$

exists in $H_{\Gamma}^{1}\left(\Omega^{+}\right)$. Note that

$$
\left.\frac{d}{d t}\left(I_{t} f \circ F_{t}, \phi\right)\right|_{t=0}=(\operatorname{div} h f, \phi)+(h \cdot \nabla f)=(\operatorname{div}(f h), \phi)=-(f, h \cdot \nabla \phi) .
$$

Since $\frac{d}{d t} A_{t}=A=\operatorname{div} h I-\left((D h)^{*}+D h\right)$, it follows from (A.9) that $\dot{u}$ satisfies

$$
(\nabla \dot{u}, \nabla \phi)+(A \nabla u, \nabla \phi)=0
$$

for all $\phi \in H_{\Gamma}^{1}\left(\Omega^{+}\right)$.

Next we derive an equation for the shape derivative $u^{\prime}$. Note that

$$
\begin{aligned}
& (\nabla(h \cdot \nabla u), \nabla \phi)+(A \nabla u, \nabla \phi)-(\Delta u, h \cdot \nabla \phi) \\
& =\left\langle\left(\begin{array}{cc}
\left(h_{2}\right)_{x_{2}}-\left(h_{1}\right)_{x_{1}} & -\left(\left(h_{1}\right)_{x_{2}}+\left(h_{2}\right)_{x_{1}}\right) \\
-\left(\left(h_{1}\right)_{x_{2}}+\left(h_{2}\right)_{x_{1}}\right) & \left(h_{1}\right)_{x_{1}}-\left(h_{2}\right)_{x_{2}}
\end{array}\right)\left(\begin{array}{c}
u_{x_{1}} \\
u_{x_{2}}
\end{array}\right),\left(\begin{array}{c}
\phi_{x_{1}} \\
\phi_{x_{2}}
\end{array}\right)\right. \\
& \left.+\left(\begin{array}{c}
\left(h_{1}\right)_{x_{1}} u_{x_{1}}+h_{1} u_{x_{1} x_{1}}+\left(h_{2}\right)_{x_{1}} u_{x_{2}}+h_{2} u_{x_{1} x_{2}}-h_{1}\left(u_{x_{1} x_{1}}+u_{x_{2} x_{2}}\right) \\
\left(h_{1}\right)_{x_{2}} u_{x_{1}}+h_{1} u_{x_{1} x_{2}}+\left(h_{2}\right)_{x_{2}} u_{x_{2}}+h_{2} u_{x_{2} x_{2}}-h_{2}\left(u_{x_{1} x_{1}}+u_{x_{2} x_{2}}\right)
\end{array}\right),\left(\begin{array}{c}
\phi_{x_{1}} \\
\phi_{x_{2}}
\end{array}\right)\right\rangle \\
& =\left(\left(h_{2} u_{x_{1}}-h_{1} u_{x_{2}}\right)_{x_{2}}, \phi_{x_{1}}\right)-\left(\left(h_{2} u_{x_{1}}-h_{1} u_{x_{2}}\right)_{x_{1}}, \phi_{x_{2}}\right) \\
& =\left(\operatorname{curl}\left(h_{2} u_{x_{1}}-h_{1} u_{x_{2}}\right), \operatorname{grad} \phi\right)=0,
\end{aligned}
$$

for $\phi \in H_{\Gamma}^{1}\left(\Omega^{+}\right)$, since $\phi_{\tau}=0$ on $\Gamma$. Thus, from (A.10)-(A.11) we obtain

$$
\left(\nabla u^{\prime}, \nabla \phi\right)=0 \quad \text { for } \phi \in H_{\Gamma}^{1}\left(\Omega^{+}\right)
$$

with boundary conditions

$$
u^{\prime}+h \cdot \nabla u=0 \quad \text { on } \Gamma \text { and } \frac{\partial u^{\prime}}{\partial \nu}=0 \text { on } \partial \Omega .
$$

Since $u_{\tau}=0$ on $\Gamma$ we have $(2.9),(2.10)$. 